zealots and we cannot effectively prepare for an attack in advance. But in reality, much can be done to prevent a nuclear strike in a major city; most importantly, nations can work harder to secure and ultimately destroy the world's stockpiles of highly enriched uranium.

As for unfolding trends, Smil includes in his list humankind's transition away from fossil fuels as a main energy source, shifting power relations among major nations, widening economic inequalities, climate change, perturbations of the global nitrogen cycle and antibiotic resistance. These trends do not sit together easily - for example, some implicate Earth's natural systems whereas others are mostly the product of human social and economic interactions - so Smil's analysis often comes across as a rather indigestible series of apples and oranges. Within the same chapter, for instance, he jumps from a treatment of the intrinsic power-density limits of renewable energy to an assessment of the geopolitics of world order.

Of greater concern is Smil's tendency to slip into polemic. His discussions of constraints on conventional oil supply and of climate change are selective and sometimes simply wrong. For example, he dismisses the possibility of abrupt climate shifts, based on a muddled interpretation of the science of millennial climate events recorded in ice cores (known as Dansgaard-Oeschger oscillations); he downplays recent research that strongly suggests sea levels will rise much faster than projected by the Intergovernmental Panel on Climate Change; and he wrongly suggests that the Gulf Stream is not driven partly by thermohaline circulation and that it does not contribute to Europe's warmth. He also repeats, as an illustration of how past predictions can be wildly wrong, the myth that scientists largely supported the early 1970s theory that global cooling was imminent.

Patten cannot claim Smil's technical expertise, but his book is more balanced. He acknowledges his biases and the limits of his knowledge, and gives fair consideration to contrary views. Some of his passages, especially those on China and India, are wonderfully rich. And his prescriptions are simultaneously deeply humane and pragmatically bounded by a long familiarity with the ways of the world - no mean feat.

Yet too often What Next? seems laboriously descriptive, absorbed in details of how we got to where we are, rather than focusing on what might happen later this century. The book's time horizon is only a few decades into the future, so its subtitle is misleading. And one is left with the disquieting sense that the statebased apparatus that Patten calls on to save humankind is not remotely up to the task. Patten is right to admire the power and potential of Western institutions, but the challenges we now face, such as climate change, are decidedly different from those that such institutions evolved to address.

This brings us to why these books ultimately disappoint: in each case the analysis, although clearly structured, is not guided by an underlying theory of societal crisis. True, such a theory would not give the authors the power to predict the future with any precision, but it would help them to identify which combinations of factors might lead humankind over the precipice, and what we might do to avoid such a fate.

Recent research has highlighted fundamental causes of societal crisis. These include the convergence of interacting stresses that overloads the coping capacity of multiple components of society, especially the state; rising social and technical complexity that generates diminishing marginal societal returns; the rapidly escalating cost of getting energy; high connectivity among people, institutions and technologies; and the declining redundancy and resilience of crucial systems.

One can make a credible argument, grounded in just the kind of evidence Smil and Patten use, that all of the above factors are now acting powerfully in humankind's tightly coupled, planetary, socio-ecological system. In the absence of a serviceable theory of societal crisis, in the end both books present little more than a laundry list of things we should worry about. For our children's and grandchildren's sake, we need much more, and fast.

Thomas Homer-Dixon is at the Balsillie School of International Affairs, Waterloo, Ontario N2L 6C2, Canada, and author of The Upside of Down: Catastrophe, Creativity and the Renewal of Civilisation.

e-mail: tad@homerdixon.com

\title{
Universe in a box
}

\section{Joseph Cornell and Astronomy: A Case for the Stars \\ by Kirsten Hoving \\ Princeton University Press: 2008 \\ 336 pp. \$49.50, £35}

Sculptor Joseph Cornell made boxes. Intricate, three-dimensional montages of photographs, sky maps and paintings, neatly packed into wooden cases full of bric-à-brac: eggs, pipes, glasses, shells, stamps, chains and whatever objects fitted with his internal logic. And, even though he was considered one of the American exponents of surrealism, that epitome of irrationality, there was a logic. In a mesmerizing, if mildly flawed, attempt to immerse us in Cornell's crafted universes, art historian Kirsten Hoving uses the artist's fascination with astronomy to tease out the logic that underlies his work.

Cornell's life was complicated. Although he was from an affluent background, he had to support his younger brother who suffered from cerebral palsy, and until the late 1940s worked variously as a salesman, textile designer and in

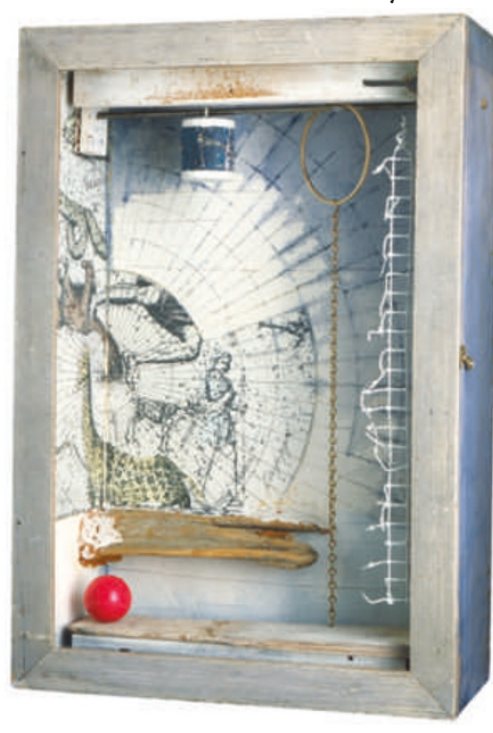

Joseph Cornell's work mirrors the 1960s US cultural fascination with astronomy. a plant nursery. He was a staunch believer in the teachings of Mary Baker Eddy, the founder of Christian Science, and never forged a longlasting relationship with a companion, spending most of his life in the same house in Utopia Parkway, a working-class neighbourhood of Flushing in Queens, New York. Yet, at the same time, his boxes and experimental films were admired in the high-octane art scene of New York, where he took part in the first surrealist exhibition at the Museum of Modern Art. Marcel Duchamp introduced him into the orbit of Peggy Guggenheim and her coterie, and from the early 1950s onwards, he was able to make a living through his art.

Hoving does something ambitious and difficult: she identifies one important thread of his creative process and uses it to help us understand Cornell's art. Astronomy clearly played an important part in his work and life. His boxes, films and the countless dossiers that he stored at his house were littered with star maps, references to Albert Einstein and Arthur Stanley Eddington, solar eclipses and his fascination with space travel. Hoving uses these obsessions to relate a body of work that spans many decades. Albeit an effective choice, astronomy is only 
one thread of many, and it competes against his fascination with the natural sciences, art, poetry, modern dance and popular culture. Is one strand enough to understand him by?

The book's relentless focus on astronomy is to the detriment of a more comprehensive view of Cornell as an artist and as a man. Very little is said about his personal life, with only fleeting references to his reclusiveness, his close relationship with his brother and mother and his romantic trysts in later life. To compensate, Hoving extends her prose, clogging up the flow of the narrative. She feels obliged to describe the minutiae of individual artworks, to pepper the text with vignettes on cosmology and astronomy, and to make the case for a few of the more tenuous links between his work and astronomy. To be fair, Hoving's arguments are on the whole convincing and at times enthralling, and her access to the debris of Cornell's life puts her in a unique position to back up many of her claims. But her thesis could have been made in half the number of pages. This is certainly a case in which less would have been more.

Yet, with its high-quality production and beautiful and wide-ranging illustrations, the book is extremely absorbing. I see it as Hoving's attempt to construct her own box, a carefully crafted piece laden with excerpts and images that penetrate Cornell's world and his obsessions. Furthermore, it is a showcase for the enthusiasm with the modern that pervaded American popular culture during the twentieth century. By including newspaper articles and advertisements of the time in which astronomy and the space race were prominent, Hoving shows that Cornell was mirroring what he saw around him.

At a time when the interplay of science and art is ever more present in cultural life, and we begin to ask ourselves whether much of what is done is any good, Joseph Cornell and Astronomy gives us a good example of what works best: quiet fascination and obsession allied with genius.

Pedro Ferreira is a professor in astrophysics at the University of Oxford, Keble Road, Oxford OX13RH, UK, and author of The State of the Universe. e-mail:p.ferreira1@physics.ox.ac.uk

\section{A romp through science fiction}

\section{Nanovision: Engineering the Future \\ by Colin Milburn \\ Duke University Press: 2008 \\ 296 pp. \$22.95, £17.99}

Nanotechnology, we are often told, will change every aspect of our lives. It is easy to hype such claims because of the long timescales for approving a new technology for commercialization. The science itself can sound like science fiction at times, and fears about how nanotechnology will be realized in the future crop up in works of fiction. In Nanovision, historian of science Colin Milburn proposes that such speculation is an integral part of nanotechnology.

Milburn examines nanotechnology in a wealth of science-fiction texts, investigating such themes as the manipulation of matter, artificial life forms and the spread of nanobots as a 'grey goo' that ultimately consumes Earth. Many writers portray the science in a negative light, as the enabler of humankind's destruction or enslavement. Other imaginative works simply exploit differences between the physical laws that rule the nanoworld and the macroworld we live in, such as the effect of surface tension on a microscopic fictional organism. Milburn also looks at individuals who have straddled the fields of science and sci-fi, including molecular engineer and futurologist Eric Drexler, and carbon-nanotube discoverer Richard Smalley, who envisioned an elevator made of nanotubes stretching from Earth into space.

The book fills gaps that most nanoscientists have in their knowledge about the history of a field that has evolved so fast as to effectively obscure its founders. However, Milburn tries too hard to prove the mix of science and fiction. In focusing on nanotechnology, he neglects the fact that forward thinking, technology forecasting and hyped claims are common to many scientific disciplines, such as genetics, energy research and space flight. More worryingly, he confuses theoretical science and fiction, missing the point that theories are not just speculation but are underpinned by mathematics, physical laws and reproducible calculations. His analysis also takes too literally the timetables and roadmaps contained in grant proposals and the like; documents that scientists consider to be indicative rather than prescriptive.

Some parts of the book, even the introduction, are difficult to follow. The writing is shrouded in the complex language of postmodern literary theory, and is often as dense as a hard-core physics paper. Milburn's references to gendered themes of male domination, even when discussing scientific instruments such as the scanning tunnelling microscope, are sometimes involuntarily comical. Yet he ignores the large number of women in nanoscience and their views: no female nanoscientists are cited in the book.

Nanovision is not the place to seek clues about where nanotechnology is going, as it contains few scientific developments past the start of this decade. Beyond the hype, many useful applications - ranging from self-cleaning surfaces to sunscreen lotion containing nanoparticles of titanium dioxide, to nanocomposite materials in the car industry - are already on the market. Furthermore, in contrast to fears that the world will be devoured by grey goo, studies have shown that public perceptions of nanotechnology are mostly positive. Although there are real and tangible risks regarding the toxicity of nanomaterials, the scaremongering that fascinates Milburn is not such a pressing concern. But if you like a romp through themes that mix current nanoscience and literature in interesting ways, Milburn's book is a valuable read. Perhaps reality is stranger than fiction.

Stefano Tonzani is materials science editor at Nature.

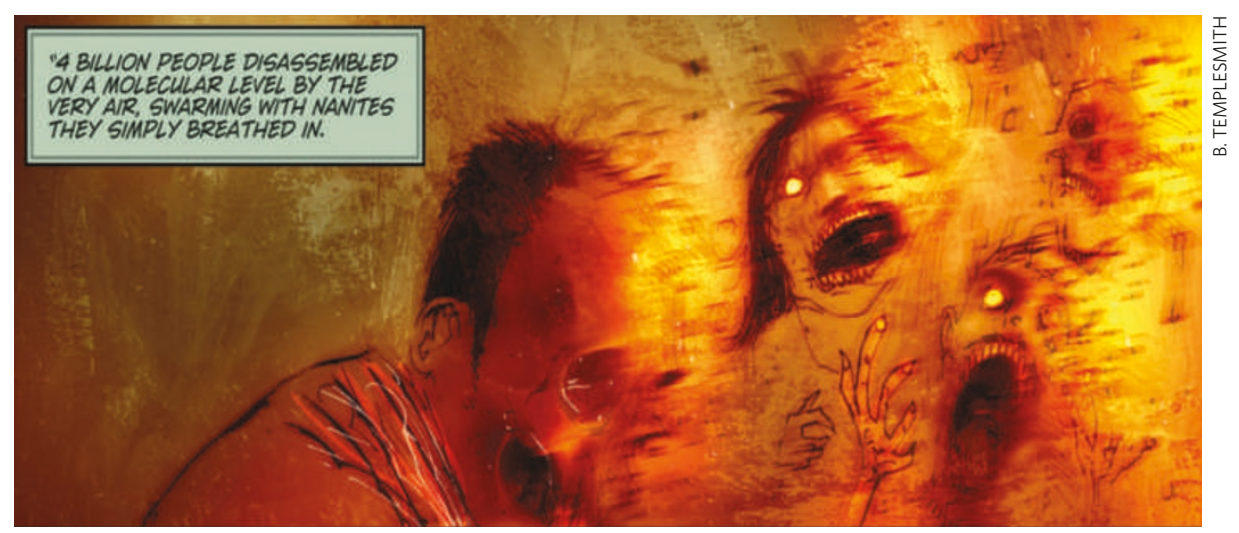

Nanotechnology is often portrayed in fiction as destructive, but public perceptions are more positive. 